\title{
Alpha power transformed extended exponential distribu- tion: properties and applications
}

\author{
Amal S. Hassana, Rokaya E. Mohamda ${ }^{a}$ M. Elgarhy, ${ }^{\mathrm{b}, *}$, Aisha Fayomic \\ a Institute of Statistical Studies and Research, Cairo University, Egypt. \\ ${ }^{b}$ Vice Presidency for Graduate Studies and Scientific Research, University of Jeddah, Jeddah, KSA. \\ ${ }^{c}$ Statistics Department, Faculty of Science, King AbdulAziz University, Jeddah, KSA.
}

\begin{abstract}
In this paper, a three-parameter lifetime model motivated by alpha power transformation is considered. We call the proposed distribution as; the alpha power transformed extended exponential (APTEE). The APTEE model contains new recent models as; alpha power transformed exponential and alpha power transformed Lindley distributions. At the same time, it contains classical models as exponential, gamma, and Lindley distributions. The properties of the APTEE distribution are derived. Parameter estimation is accomplished using maximum likelihood, percentiles, and Cramer-von Mises methods. Simulation issues and applications to real data are emphasized.
\end{abstract}

Keywords: Extended exponential, moments, maximum likelihood, percentiles and Cramer-von Mises.

2010 MSC: 60E05, 62E10, 62N05.

(C)2019 All rights reserved.

\section{Introduction}

Modeling lifetime data is important and crucial in many fields such as medicine, engineering, demography, etc.. So, diverse lifetime distributions have been introduced, in the probability theory, to model specific real life data. In recent statistical researches, diverse useful processes of extending and developing new continuous distributions were established to yield new models.

Recently, the modified and extended forms of the exponential distribution were proposed by several authors. For example, the generalized exponential (GE) distribution was suggested by Gupta and Kundu [8, 9] as a generalized form of the exponential distribution. Further, Nadarajah and Haghighi [17] introduced another extension of the exponential model. The probability density function (pdf) of a random variable $X$ having the Nadarajah and Haghighi's exponential (NHE) distribution is given by

$$
h_{\mathrm{NHE}}(x ; \beta, \gamma)=\gamma \beta(1+\gamma x) e^{1-(1+\gamma x)^{\beta}}, x, \gamma, \beta>0 .
$$

\footnotetext{
*Corresponding author

Email addresses: dr.amalelmoslamy@gmail.com (Amal S. Hassan), rokayaelmorsy@gmail.com (Rokaya E. Mohamd),

m_elgarhy85@yahoo.com (M. Elgarhy), af ayomi@kau.edu.sa (Aisha Fayomi)

doi: $10.22436 /$ jnsa.012.04.05
}

Received: 2018-09-17 Revised: 2018-10-10 Accepted: 2018-10-15 
The Kumaraswamy exponential, based on Kumaraswamy generated family, has been suggested by Cordeiro and de Castro [2]. Ristic and Balakrishnan [21] proposed the gamma exponentiated exponential distribution. Merovci [16] introduced transmuted exponentiated exponential distribution. An extended exponential (EE) distribution has been suggested by Gómez et al. [7], depending on the extension of Nadarajah and Haghighi [17], with the following cumulative distribution function (cdf) and pdf

$$
\mathrm{H}_{\mathrm{EE}}(x ; \gamma, \beta)=\frac{\gamma+\beta-(\gamma+\beta+\gamma \beta x) e^{-\gamma x}}{\gamma+\beta}, x, \gamma, \beta>0,
$$

and

$$
h_{E E}(x ; \gamma, \beta)=\frac{\gamma^{2}(1+\beta x) e^{-\gamma x}}{\gamma+\beta}, x, \gamma, \beta>0 .
$$

The exponentiated generalized EE and the modified exponential distributions have been proposed, respectively, by de Andrade et al. [5] and Rasekhi et al. [19].

Recently, alpha power transformation (APT) has been proposed by Mahdavi and Kundu [15] in order to get more flexibility to a family of distributions. The cdf of a continuous random variable $X$ has APT family is defined as follows:

$$
\mathrm{H}_{\mathrm{APT}}(\mathrm{x} ; \alpha)= \begin{cases}\frac{\alpha^{\mathrm{H}(x)}-1}{\alpha-1}, & \alpha>0, \alpha \neq 1 \\ \mathrm{H}(\mathrm{x}), & \alpha>0, \alpha=1\end{cases}
$$

In view of the APT, we explore the APTEE model from the EE distribution. The APTEE distribution contains special recent models, namely; APT exponential (Mahdavi and Kundu [15]) and APT Lindley (Dey et al. [6]). At the same time, it contains special classical distributions which are exponential, Lindley and gamma. Statistical properties are implemented. Estimation of the parameters and applications with real data are given. This paper is constructed as follows. In Sections 2 and 3, we study the APTEE, and explore its properties. In Section 4, we obtain the maximum likelihood (ML), percentiles and Cramer- vonMises estimators and their effectiveness are examined via a numerical study. The analyses of two real data sets are employed in Section 5. The paper ends with concluding remarks.

\section{The APTEE model}

The distribution function of APTEE distribution with set of parameters $\Phi=(\alpha, \beta, \gamma)$ is obtained by substituting the cdf (1.1) in (1.2) as follows

$$
\mathrm{H}_{\text {APTEE }}(x ; \varpi)=\left[\begin{array}{ll}
\frac{\alpha^{\frac{\gamma+\beta-(\gamma+\beta+\gamma \beta x) e^{-\gamma x}}{\gamma+\beta}}-1}{\alpha-1}, & x, \beta, \gamma, \alpha>0, \alpha \neq 1, \\
\frac{\gamma+\beta-(\gamma+\beta+\gamma \beta x) e^{-\gamma x}}{\gamma+\beta}, & x, \beta, \gamma, \alpha>0, \alpha=1 .
\end{array}\right.
$$

The pdf of APTEE distribution is given by

$$
h_{\text {APTEE }}(x ; \varpi)=\left[\begin{array}{ll}
\frac{\log \alpha \gamma^{2}(1+\beta x) e^{-\gamma x} \alpha^{\frac{\gamma+\beta-(\gamma+\beta+\gamma \beta x) e^{-\gamma x}}{\gamma+\beta}}}{\alpha-1} \frac{}{\gamma+\beta}, & x, \beta, \gamma>0, \alpha \neq 1, \\
\frac{\gamma^{2}(1+\beta x) e^{-\gamma x}}{\gamma+\beta}, & x, \alpha, \beta, \gamma>0, \alpha=1 .
\end{array}\right.
$$

Also, the reliability function, say $\overline{\mathrm{H}}_{\mathrm{APTEE}}(\mathrm{x} ; \varpi)$, and hazard rate function $(\mathrm{hrf})$, say $\Xi_{\mathrm{APTEE}}(\mathrm{x} ; \varpi)$ of $X$ are given, respectively, as follows:

$$
\bar{H}_{\text {APTEE }}(x ; \varpi)=\left[\begin{array}{ll}
\frac{\alpha-\alpha \frac{\gamma+\beta-(\gamma+\beta+\gamma \beta x) e^{-\gamma x}}{\gamma+\beta}}{\alpha-1}, & x, \beta, \gamma, \alpha>0, \alpha \neq 1, \\
\frac{(\gamma+\beta+\gamma \beta x) e^{-\gamma x}}{\gamma+\beta}, & x, \beta, \gamma, \alpha>0, \alpha=1
\end{array}\right.
$$


and

$$
\Xi_{\text {APTEE }}(x ; \varpi)=\left[\begin{array}{ll}
\frac{\log (\alpha) \gamma^{2} \alpha^{\frac{\gamma+\beta-(\gamma+\beta+\gamma \beta x) e^{-\gamma x}}{\gamma+\beta}}(1+\beta x) e^{-\gamma x}}{(\gamma+\beta)\left\{\alpha-\alpha \frac{\gamma+\beta-(\gamma+\beta+\gamma \beta x) e^{-\gamma x}}{\gamma+\beta}\right\}}, & x, \beta, \gamma, \alpha>0, \alpha \neq 1, \\
\frac{\gamma^{2}(1+\beta x)}{\gamma+\beta+\gamma \beta x}, & x, \beta, \gamma, \alpha>0, \alpha=1 .
\end{array}\right.
$$

Special sub-models of the APTEE distribution are recorded in Table 1.

Table 1: Sub-models of the APTEE distribution.

\begin{tabular}{|c|c|c|c|}
\hline B & $\alpha$ & Reduced model & Authors \\
\hline 0 & - & APT exponential (APTE) & Mahdavi and Kundu [15] \\
\hline 1 & - & APT Lindley (APTL) & Dey et al. [6] \\
\hline 0 & 1 & Exponential & \\
\hline 1 & 1 & Lindley & Lindley [11] \\
\hline 1 & 1 & Gamma $(2, \gamma)$ & \\
\hline
\end{tabular}

Hereafter, a random variable $X$ that follows the distribution in (2.1) is denoted by $X \sim$ APTEE $(\varpi)$. Some descriptive pdf and hrf plots of $X \sim$ APTEE $(\varpi)$ are illustrated below for specific parameter choices of $\varpi$ (see Figure 1).

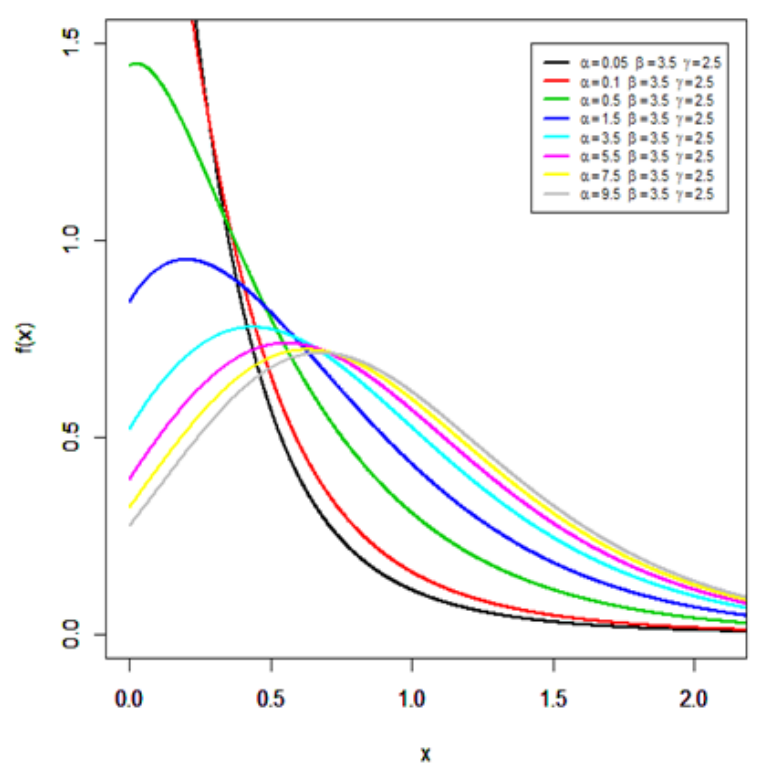

Figure 1: Plots of the pdf of the APTEE distribution.

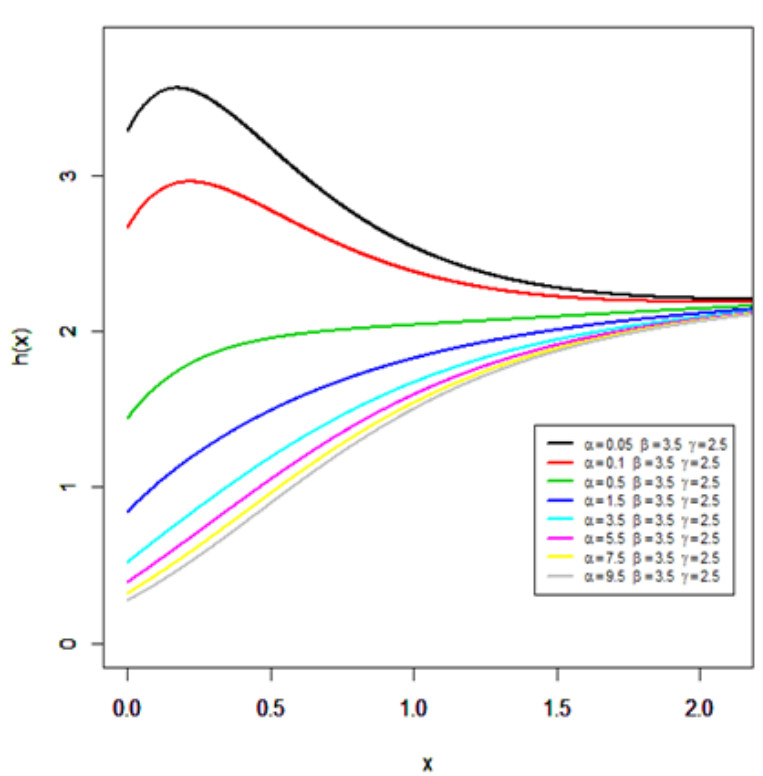

Figure 2: Plots of the hrf the APTEE distribution.

From Figure 1, we conclude that pdf of APTEE distribution can be reversed J-shaped, uni-model and right skewed. Also, the hrf of APTEE distribution can be increasing and decreasing as seen from Figure 2.

\section{Statistical properties}

\subsection{Quntile function}

The APTEE distribution can be easily simulated by inverting cdf (2.1) as follows: if $p$ follows uniform distribution on $(0,1)$, then

$$
\mathrm{Q}(\mathrm{p})=-\frac{1}{\beta}-\frac{1}{\gamma}-\frac{1}{\gamma} \mathrm{W}_{-1}\left[-\frac{1}{\beta}(\gamma+\beta)\left\{\frac{1-\log [\mathrm{p}(\alpha-1)+1]}{\log \alpha}\right\} e^{-\left(\frac{\gamma+\beta}{\beta}\right)}\right],
$$


where $W($.$) is the Lambert W$-function and $0<p<1$. According to Corless et al. [3] and Jodra [10]) the Lambert $W$ function has been applied to solve several problems in mathematics, physics and engineering. Using the Lagrange inversion theorem, the power series for the $W$-function can be holed (see de Andrade et al. [5]) as follows

$$
W(t)=\sum_{m=1}^{\infty} \frac{(-m)^{m-1} t^{m}}{m !}
$$

From (3.1) and (3.2), we have

$$
\mathrm{Q}(\mathrm{p})=-\frac{1}{\beta}-\frac{1}{\gamma}-\frac{1}{\gamma} \sum_{m=1}^{\infty} \frac{(-1)^{\mathrm{m}}(-\mathrm{m})^{\mathrm{m}-1}}{\mathrm{~m} !} \frac{(\gamma+\beta)^{\mathrm{m}}}{\beta^{m}}\left\{\frac{1-\log [\mathrm{p}(\alpha-1)+1]}{\log \alpha}\right\}^{\mathrm{m}} e^{-\mathrm{m}\left(\frac{\gamma+\beta}{\beta}\right)} .
$$

Table 2 displays the percentage points of some specific choices of the parameters. It contains the first quartile, median and the third quartile.

Table 2: Percentage points for $\gamma, \alpha$, and $\beta$.

\begin{tabular}{|c|c|c|c|c|c|c|c|c|c|c|}
\hline \multirow{2}{*}{$\gamma$} & \multirow{2}{*}{$\alpha$} & \multicolumn{3}{|c|}{$\beta=0.5$} & \multicolumn{3}{c|}{$\beta=1$} & \multicolumn{3}{c|}{$\beta=1.5$} \\
\cline { 2 - 11 } & & $25 \%$ & $50 \%$ & $75 \%$ & $25 \%$ & $50 \%$ & $75 \%$ & $25 \%$ & $50 \%$ & $75 \%$ \\
\hline \multirow{4}{*}{0.8} & 0.5 & 0.419 & 1.008 & 2.021 & 0.533 & 1.207 & 2.296 & 0.616 & 1.328 & 2.444 \\
\cline { 2 - 10 } & 2 & 0.743 & 1.600 & 2.845 & 0.910 & 1.849 & 3.154 & 1.018 & 1.989 & 3.313 \\
\cline { 2 - 10 } & 5 & 1.056 & 2.045 & 3.357 & 1.260 & 2.320 & 3.681 & 1.383 & 2.469 & 3.846 \\
\cline { 2 - 10 } & 10 & 1.324 & 2.370 & 3.702 & 1.552 & 2.661 & 4.035 & 1.685 & 2.815 & 4.202 \\
\hline \multirow{4}{*}{1.2} & 0.5 & 0.248 & 0.609 & 1.249 & 0.308 & 0.724 & 1.423 & 0.356 & 0.805 & 1.531 \\
\cline { 2 - 10 } & 2 & 0.445 & 0.980 & 1.778 & 0.538 & 1.134 & 1.983 & 0.607 & 1.233 & 2.103 \\
\cline { 2 - 10 } & 5 & 0.639 & 1.264 & 2.110 & 0.758 & 1.439 & 2.330 & 0.840 & 1.547 & 2.454 \\
\cline { 2 - 9 } & 10 & 0.806 & 1.472 & 2.335 & 0.944 & 1.661 & 2.563 & 1.035 & 1.774 & 2.690 \\
\hline
\end{tabular}

We detect from Table 1 that as the value of $\alpha$ increases, for fixed values of $\beta$ and $\gamma$, the value of percentage points increases. As the value of $\gamma$ increases, for fixed values of $\alpha$ and $\beta$, the percentage value points decreases. Also, as the value of $\beta$ increases, for fixed values of $\alpha$ and $\gamma$, the percentage value points decreases.

\subsection{Moments}

In this subsection, the $\mathrm{r}^{\text {th }}$ moment and moment generating function (mgf) of APTEE distribution are derived. Using the following series representation in pdf (2.2)

$$
\alpha^{w}=\sum_{i=0}^{\infty} \frac{(\log \alpha)^{i}}{i !} w^{i},
$$

hence, the $r^{\text {th }}$ moment of APTEE distribution can be formed as follows

$$
\mu_{r}^{\prime}=\frac{\gamma^{2}}{\gamma+\beta} \sum_{i=0}^{\infty} \frac{(\log \alpha)^{i+1}}{(\alpha-1) i !} \int_{0}^{\infty} x^{r}(1+\beta x) e^{-\gamma x}\left[\frac{\gamma+\beta-(\gamma+\beta+\gamma \beta x) e^{-\gamma x}}{\gamma+\beta}\right]^{i} d x .
$$

Using the binomial expansion, then

$$
\mu_{r}^{\prime}=\frac{\gamma^{2}}{\gamma+\beta} \sum_{i=0}^{\infty} \sum_{l=0}^{i}(-1)^{l} \frac{(\log \alpha)^{i+1}}{(\alpha-1) i !}\left(\begin{array}{l}
i \\
l
\end{array}\right) \int_{0}^{\infty} x^{r}(1+\beta x) e^{-\gamma x(l+1)}\left(\frac{\gamma+\beta+\gamma \beta x}{\gamma+\beta}\right)^{l} d x .
$$

Using the binomial another time, then the $r^{\text {th }}$ moment of APTEE distribution is

$$
\mu_{r}{ }^{\prime}=\sum_{i=0}^{\infty} \sum_{l=0}^{i} \sum_{s=0}^{l}(-1)^{l} \frac{\gamma^{2+s} \beta^{s}}{(\gamma+\beta)^{s+1}} \frac{(\log \alpha)^{i+1}}{(\alpha-1) i !}\left(\begin{array}{l}
i \\
l
\end{array}\right)\left(\begin{array}{l}
l \\
s
\end{array}\right)\left[\frac{\Gamma(r+s+1)}{\{\gamma(l+1)\}^{r+s+1}}+\frac{\beta \Gamma(r+s+2)}{\{\gamma(l+1)\}^{r+s+2}}\right]
$$

for $r=1,2, \ldots$ 
Individually, the first four moments are obtained by setting $r=1,2,3$, and 4 in (3.4). Also, the $r^{\text {th }}$ central moment $\left(\mu_{r}\right)$ of $X$ is given by

$$
\mu_{r}=E\left(X-\mu_{1}^{\prime}\right)^{r}=\sum_{i=0}^{r}(-1)^{i}\left(\begin{array}{l}
r \\
i
\end{array}\right)\left(\mu_{1}^{\prime}\right)^{i} \mu_{r-i}^{\prime} .
$$

The skewness (SK) and kurtosis (Ku) are defined by

$$
\mathrm{SK}=\frac{\mu_{3}}{\mu_{2}^{3 / 2}}, \mathrm{Ku}=\frac{\mu_{4}}{\mu_{2}^{2}} .
$$

Table 3 contains values of mean $\left(\mu_{1}^{\prime}\right)$, variance $\left(\sigma^{2}\right)$, SK, and Ku of APTEE distribution for some certain values of parameters.

\begin{tabular}{|c|c|c|c|c|c|c|c|c|c|c|c|c|c|}
\hline \multirow[b]{2}{*}{$\gamma$} & \multirow[b]{2}{*}{$\alpha$} & \multicolumn{4}{|c|}{$\beta=0.5$} & \multicolumn{4}{|c|}{$\beta=1$} & \multicolumn{4}{|c|}{$\beta=1.5$} \\
\hline & & $\mu_{1}^{\prime}$ & $\sigma^{2}$ & SK & Ku & $\mu_{1}^{\prime}$ & $\sigma^{2}$ & SK & $K u$ & $\mu_{1}^{\prime}$ & $\sigma^{2}$ & SK & $K u$ \\
\hline \multirow{4}{*}{0.8} & 0.5 & 0.633 & 1.445 & 2.894 & 14.231 & 0.718 & 1.708 & 2.67 & 12.387 & 0.766 & 1.852 & 2.551 & 11.489 \\
\hline & 2 & 0.878 & 2.265 & 2.292 & 9.483 & 0.979 & 2.637 & 2.128 & 8.406 & 1.035 & 2.835 & 2.043 & 7.888 \\
\hline & 5 & 1.05 & 2.859 & 1.99 & 7.578 & 1.161 & 3.304 & 1.851 & 6.773 & 1.221 & 3.539 & 1.779 & 6.39 \\
\hline & 10 & 1.176 & 3.307 & 1.805 & 6.559 & 1.294 & 3.806 & 1.681 & 5.893 & 1.356 & 4.067 & 1.617 & 5.578 \\
\hline \multirow{4}{*}{1.2} & 0.5 & 0.393 & 0.579 & 3.015 & 15.332 & 0.445 & 0.69 & 2.801 & 13.436 & 0.478 & 0.759 & 2.67 & 12.387 \\
\hline & 2 & 0.549 & 0.914 & 2.383 & 10.137 & 0.613 & 1.076 & 2.224 & 9.016 & 0.653 & 1.172 & 2.128 & 8.406 \\
\hline & 5 & 0.659 & 1.158 & 2.067 & 8.071 & 0.731 & 1.354 & 1.931 & 7.228 & 0.774 & 1.469 & 1.851 & 6.773 \\
\hline & 10 & 0.739 & 1.343 & 1.876 & 6.97 & 0.817 & 1.563 & 1.753 & 6.269 & 0.863 & 1.692 & 1.681 & 5.893 \\
\hline
\end{tabular}

Table 3: $\mu_{1}^{\prime}, \sigma^{2}, \mathrm{Sk}$, and $\mathrm{Ku}$ of APTEE distribution.

From Table 3, we conclude that, as the values of $\alpha$ and $\beta$ increase then the values of $\mu_{1}^{\prime}$ and $\sigma^{2}$ are increasing, whereas, the values of SK and $K u$ are decreasing. As the values of $\alpha$ and $\gamma$ increase then the values of $\mu_{1}^{\prime}$ and $\sigma^{2}$ are decreasing, whereas, the values of $S K$ and $K u$ are increasing. Also, we conclude that the distribution is skewed to right and leptokurtic.

Furthermore, the mgf of APTEE distribution is given by

$$
M_{X}(t)=\sum_{r, i=0}^{\infty} \sum_{l=0}^{i} \sum_{s=0}^{l}(-1)^{l} \frac{t^{r}}{r !} \frac{\gamma^{2+s} \beta^{s}}{(\gamma+\beta)^{s+1}} \frac{(\log \alpha)^{i+1}}{(\alpha-1) i !}\left(\begin{array}{l}
i \\
l
\end{array}\right)\left(\begin{array}{l}
l \\
s
\end{array}\right)\left[\frac{\Gamma(r+s+1)}{\{\gamma(l+1)\}^{r+s+1}}+\frac{\beta \Gamma(r+s+2)}{\{\gamma(l+1)\}^{r+s+2}}\right] \text {. }
$$

\subsection{Moments of residual life}

The $\mathrm{m}^{\text {th }}$ moment of the residual life of APTEE distribution is obtained by using expansion (3.3) as follows

$\pi_{\mathrm{m}}(\mathrm{t})=\frac{1}{\overline{\mathrm{H}}_{\mathrm{APTEE}}(\mathrm{x} ; \varpi)} \frac{\gamma^{2}}{\gamma+\beta} \sum_{i=0}^{\infty} \frac{(\log \alpha)^{i+1}}{(\alpha-1) i !} \int_{t}^{\infty}(x-t)^{\mathrm{m}}(1+\beta x) e^{-\gamma x}\left[\frac{\gamma+\beta-(\gamma+\beta+\gamma \beta x) e^{-\gamma x}}{\gamma+\beta}\right]^{i} \mathrm{~d} x$.

Then by using the binomial expansions, several times, we obtain

$$
\begin{aligned}
\pi_{\mathrm{m}}(\mathrm{t})= & \frac{1}{\overline{\mathrm{H}}_{\text {APTEE }}(x ; \varpi)} \sum_{i=0}^{\infty} \sum_{l=0}^{i} \sum_{s=0}^{m} \sum_{k=0}^{l}(-1)^{l+m-s} t^{m-s} \frac{\gamma^{2+k} \beta^{k}}{(\gamma+\beta)^{k+1}} \frac{(\log \alpha)^{i+1}}{(\alpha-1) i !}\left(\begin{array}{c}
i \\
l
\end{array}\right)\left(\begin{array}{c}
m \\
s
\end{array}\right)\left(\begin{array}{c}
l \\
k
\end{array}\right) \\
& \times\left[\frac{\Pi(s+k+1, \gamma(\ell+1))}{\{\gamma(\ell+1)\}^{m+1}}+\frac{\beta \Pi(s+k+2, \gamma(\ell+1)}{\{\gamma(\ell+1)\}^{m+2}}\right],
\end{aligned}
$$

where, $\Pi(.,$.$) is the upper incomplete gamma function. Also, the mean residual life of APTEE distribution$ can be derived by subsituting $\mathrm{m}=1$ in the previous equation. 


\subsection{The probability weighted moments}

For a random variable $X$, the class of probability-weighted moments (PWMs), denoted by $\eta_{r, q}$, is defined as follows

$$
\eta_{r, q}=E\left[X^{r} F(x)^{q}\right]=\int_{-\infty}^{\infty} x^{r} f(x)(F(x))^{q} d x
$$

The PWM of APTEE distribution is derived by inserting (2.1) and (2.2) into (3.5), as follows

$$
\eta_{r, q}=\int_{0}^{\infty} x^{r} \frac{\log \alpha}{\alpha-1} \frac{\gamma^{2}(1+\beta x) e^{-\gamma x} \alpha^{\frac{\gamma+\beta-(\gamma+\beta+\gamma \beta x) e^{-\gamma x}}{\gamma+\beta}}}{\gamma+\beta}\left[\frac{\alpha^{\frac{\gamma+\beta-(\gamma+\beta+\gamma \beta x) e^{-\gamma x}}{\gamma+\beta}}-1}{\alpha-1}\right]^{\mathrm{q}} \mathrm{d} x .
$$

Using the binomial expansion, then

$$
\eta_{r, q}=\frac{\gamma^{2} \log \alpha}{(\gamma+\beta)(\alpha-1)^{q+1}} \sum_{k=0}^{q}(-1)^{q-k}\left(\begin{array}{l}
q \\
k
\end{array}\right) \int_{0}^{\infty} x^{r} \alpha^{(q+1)\left(\frac{\gamma+\beta-(\gamma+\beta+\gamma \beta x)-\gamma x}{\gamma+\beta}\right)}(1+\beta x) e^{-\gamma x} d x .
$$

Using (3.3) and binomial expansion, then (3.6) will be

$$
\begin{aligned}
\eta_{r, q}= & \sum_{k=0}^{q} \sum_{i=0}^{\infty} \sum_{l=0}^{i} \sum_{s=0}^{l}(-1)^{l+q-k} \frac{(\log \alpha)^{i+1} \gamma^{2+s} \beta^{s}}{(\alpha-1)^{q+1} i !(\gamma+\beta)^{s+1}}(q+1)^{i}\left(\begin{array}{l}
i \\
l
\end{array}\right)\left(\begin{array}{l}
q \\
k
\end{array}\right)\left(\begin{array}{l}
l \\
s
\end{array}\right) \\
& \times\left[\frac{\Gamma(r+s+1)}{\{\gamma(l+1)\}^{r+s+1}}+\frac{\beta \Gamma(r+s+2)}{\{\gamma(l+1)\}^{r+s+2}}\right] .
\end{aligned}
$$

\subsection{Rényi Entropy}

The entropy of a random variable provides an excellent gadget to quantify the amount of information (or uncertainty) contained in a random observation regarding its parent distribution (population). A large value of entropy implies the greater uncertainty in the data (Rényi [20]). The concept of entropy is crucial in various situations in science, engineering and economics. The Rényi entropy of a random variable $X$, for $v>0$, and $v \neq 1$, is defined by

$$
I_{R}(x)=(1-v)^{-1} \log \left(\int_{0}^{\infty}(f(x))^{v} d x\right) .
$$

The Rényi entropy of the APTEE distribution is obtained by inserting the pdf (2.2) in (3.7) as follows

$$
I_{R}(x)=(1-v)^{-1}\left(\frac{\gamma^{2} \log \alpha}{(\alpha-1)(\gamma+\beta)}\right)^{v} \log \int_{0}^{\infty}(1+\beta x)^{v} e^{-\gamma v x} \alpha^{v\left[\frac{\gamma+\beta-(\gamma+\beta+\gamma \beta x) e^{-\gamma x}}{\gamma+\beta}\right]} d x
$$

From (3.3), then $I_{R}(x)$ will be reduced to

$$
I_{R}(x)=(1-v)^{-1}\left(\frac{\gamma^{2} \log \alpha}{(\alpha-1)(\gamma+\beta)}\right)^{v} \sum_{i=0}^{\infty} \frac{(v \log \alpha)^{i}}{i !} \log \left\{\int_{0}^{\infty}(1+\beta x)^{v} e^{-\gamma x v}\left[1-\left(1+\frac{\gamma \beta x}{\gamma+\beta}\right) e^{-\gamma x}\right]^{i} d x\right\}
$$

Using the binomial expansion, more than one time, then the Rényi entropy of APTEE distribution is

$$
\begin{aligned}
\mathrm{I}_{\mathrm{R}}(\mathrm{x})= & (1-v)^{-1} \log \left\{\sum_{i=0}^{\infty} \sum_{\mathrm{l}=0}^{i} \sum_{s=0}^{\mathrm{l}} \sum_{\mathrm{m}=0}^{v}(-1)^{\mathrm{l}}\left(\frac{\log \alpha}{(\alpha-1)}\right)^{v} \frac{(\beta)^{s+v} \gamma^{s+2 v}(v \log \alpha)^{i}}{(\gamma+\beta)^{s+v} i !}\left(\begin{array}{c}
i \\
l
\end{array}\right)\left(\begin{array}{c}
l \\
s
\end{array}\right)\left(\begin{array}{c}
v \\
m
\end{array}\right)\right. \\
& \left.\times \log \left[\frac{\Gamma(s+v+1)}{(\gamma(v+l))^{s+v+1}}\right]\right\} .
\end{aligned}
$$




\subsection{Stochastic ordering}

Stochastic ordering has been recognized as a useful tool in reliability theory and other fields to assess comparative behavior. Let $X_{1}$ and $X_{2}$ be two random variables having cdfs, reliability functions and pdfs $\mathrm{H}_{\mathrm{APTEE}_{1}}\left(x ; \varpi_{1}\right)$ and $\mathrm{H}_{\mathrm{APTEE}_{2}}\left(x ; \varpi_{2}\right), \overline{\mathrm{H}}_{\mathrm{APTEE}_{1}}\left(x ; \varpi_{1}\right)$ and $\bar{H}_{\mathrm{APTEE}_{2}}\left(x ; \varpi_{2}\right)$ and $h_{\mathrm{APTEE}_{1}}\left(x ; \varpi_{1}\right)$ and $h_{\mathrm{APTEE}_{2}}\left(x ; \varpi_{2}\right)$, respectively, where $\Phi_{1}=\left(\alpha_{1}, \beta_{1}, \gamma_{1}\right)$ and $\varpi_{2}=\left(\alpha_{2}, \beta_{2}, \gamma_{2}\right)$. The random variable $X_{1}$ is said to be smaller than $X_{2}$ in the following ordering, if the following holds.

1. Likelihood ratio order $\left(X_{1} \leqslant \operatorname{lr} X_{2}\right)$ if $\frac{h_{\mathrm{APTEE}_{2}}\left(x ; \varpi_{2}\right)}{h_{\mathrm{APTEE}_{1}}\left(x ; \varpi_{1}\right)}$ is increasing in $x$.

2. Stochastic order $\left(X_{1} \leqslant s r X_{2}\right)$ if $\bar{H}_{\mathrm{APTEE}_{1}}\left(x ; \varpi_{1}\right) \leqslant \overline{\mathrm{H}}_{\mathrm{APTEE}_{2}}\left(x ; \varpi_{2}\right)$ for all $\chi$.

3. Hazard rate order $\left(X_{1} \leqslant h r X_{2}\right)$ if $\frac{\bar{H}_{\mathrm{APTEE}_{2}}\left(x ; \varpi_{2}\right)}{\overline{\mathrm{APTEE}}_{1}\left(x ; \varpi_{1}\right)}$ is increasing in $x$.

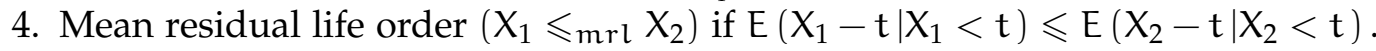

Confirming to Shaked and Shanthikumar [22], the above stochastic orders are related to each other and the following implications hold:

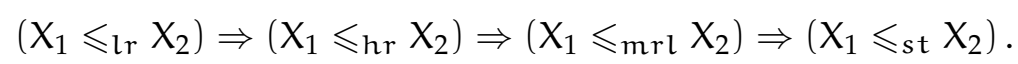

The following theorem affirms that the APTEE distribution is ordered owing to strongest likelihood ratio ordering when appropriate assumptions are satisfied.

Theorem 3.1. Let $X_{1} \sim$ APTEE $\left(\Phi_{1}\right)$ and $X_{2} \sim$ APTEE $\left(\Phi_{2}\right)$. If $\alpha_{1}=\alpha_{2}=\alpha, \beta_{1} \geqslant \beta_{2}$, and $\gamma_{1} \geqslant \gamma_{2}$, then

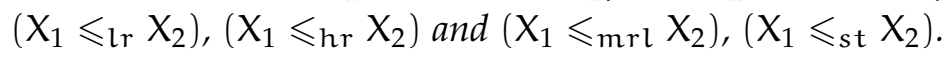

Proof. The likelihood ratio order is

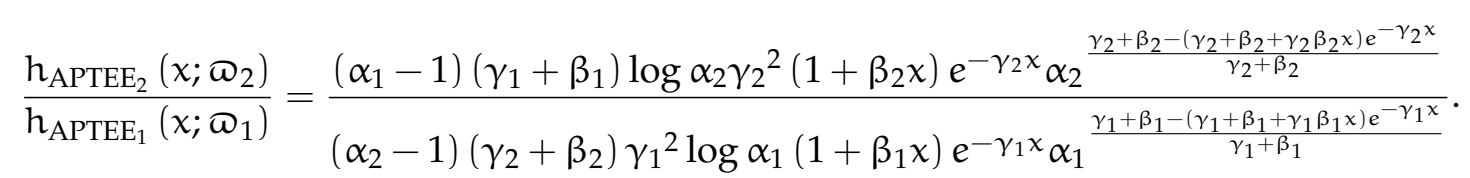

So,

$$
\begin{aligned}
\frac{d}{d x} \log \left[\frac{h_{\mathrm{APTEE}_{2}}\left(x ; \varpi_{2}\right)}{h_{\mathrm{APTEE}_{1}}\left(x ; \varpi_{1}\right)}\right]= & \frac{\beta_{2}}{\left(1+\beta_{2} x\right)}-\frac{\beta_{1}}{\left(1+\beta_{1} x\right)}+\left(\gamma_{1}-\gamma_{2}\right) x-\gamma_{2} e^{-\gamma_{2} x}\left(1+\frac{\gamma_{2} \beta_{2} x}{\gamma_{2}+\beta_{2}}\right) \log \alpha_{2} \\
& -\left(\frac{\gamma_{2} \beta_{2} e^{-\gamma_{2} x}}{\gamma_{2}+\beta_{2}}\right) \log \alpha_{2}+\gamma_{1} e^{-\gamma_{1} x}\left(1+\frac{\gamma_{1} \beta_{1} x}{\gamma_{1}+\beta_{1}}\right) \log \alpha_{1}-\left(\frac{\gamma_{1} \beta_{1} e^{-\gamma_{1} x}}{\gamma_{1}+\beta_{1}}\right) \log \alpha_{1} .
\end{aligned}
$$

Now if $\alpha_{1}=\alpha_{2}=\alpha, \beta_{1} \geqslant \beta_{2}$, and $\gamma_{1} \geqslant \gamma_{2}$, then $\frac{d}{d x} \log \left[\frac{h_{\mathrm{APTEE}_{2}}\left(x ; \Phi_{2}\right)}{h_{\mathrm{APTEE}_{1}}\left(x ; \Phi_{1}\right)}\right] \geqslant 0$, which implies that $X_{1}$ is stochastically smaller than $X_{2}$ with respect to likelihood ratio order. Similarly, we can conclude for

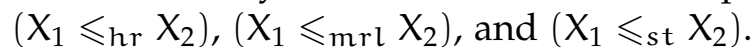

\section{Parameter estimation}

Here, estimators of population parameters are worked out via the ML, percentiles (PR), and Cramervon-Mises (CV) methods of estimation.

\subsection{Maximum likelihood estimators}

The ML estimators of the population parameters for the APTEE distribution are obtained. Let $X_{1}, \ldots, X_{n}$ be values from the APTEE distribution with set of parameters $\varpi=\{\alpha, \beta, \gamma\}^{\top}$. The log-likelihood function for the vector of parameters, say $\ell$, can be written as

$$
\ell=n \log \left[\frac{\log \alpha}{(\alpha-1)}\right]+2 n \log \gamma-n \log (\beta+\gamma)+\sum_{i=1}^{n} \log \left(1+\beta x_{i}\right)-\gamma \sum_{i=1}^{n} x_{i}
$$




$$
+\log \alpha \sum_{i=1}^{n}\left\{\frac{\gamma+\beta-(\gamma+\beta+\gamma \beta x) e^{-\gamma x}}{\gamma+\beta}\right\} .
$$

Therefore, the ML equations are given by

$$
\begin{aligned}
& \frac{\partial \ell}{\partial \alpha}=\frac{n[\alpha-1-\alpha \log \alpha]}{\alpha(\alpha-1) \log \alpha}+\frac{1}{\alpha} \sum_{i=1}^{n}\left\{\frac{\gamma+\beta-(\gamma+\beta+\gamma \beta x) e^{-\gamma x}}{\gamma+\beta}\right\}, \\
& \frac{\partial \ell}{\partial \gamma}=\frac{2 n}{\gamma}-\frac{n}{\beta+\gamma}-\sum_{i=1}^{n} x_{i}+\log \alpha \sum_{i=1}^{n} x_{i} e^{-\gamma x_{i}}\left[\frac{\gamma \beta x_{i}}{\gamma+\beta}-\frac{\beta^{2} x_{i}}{(\gamma+\beta)^{2}}\right],
\end{aligned}
$$

and

$$
\frac{\partial \ell}{\partial \beta}=\frac{-n}{\beta+\gamma}+\sum_{i=1}^{n} \frac{x_{i}}{\left(1+\beta x_{i}\right)}-\log \alpha \sum_{i=1}^{n} \frac{\gamma^{2} e^{-\gamma x_{i}}}{(\gamma+\beta)^{2}} .
$$

ML estimators of the model parameters are determined by solving numerically the non-linear equations $\partial \ell / \partial \alpha=0, \partial \ell / \partial \beta=0$, and $\partial \ell / \partial \gamma=0$ simultaneously by using mathematical package.

\subsection{Percentile estimator (PE)}

Let $X_{1}, \ldots, X_{n}$ be a random sample from the APTEE distribution and Let $X_{(1)}<X_{(2)}<\cdots<X_{(n)}$ be the corresponding order statistics. Based on PR method of estimation; the estimators of set of parameters $\varpi=\{\alpha, \beta, \gamma\}^{\top}$, are attained by minimizing the following

$$
\sum_{i=1}^{n}\left[\ln \left(p_{i}\right)-\ln \left\{\frac{\alpha^{\frac{\gamma+\beta-\left(\gamma+\beta+\gamma \beta x_{(i)}\right) e^{-\gamma x_{(i)}}}{\gamma+\beta}}-1}{\alpha-1}\right\}\right]^{2},
$$

with respect to $\varpi$, where $p_{i}$ denotes some estimates of $H_{\text {APTEE }}\left(x_{(i)} ; \varpi\right)$, and $p_{i}=i / n+1$.

\subsection{The Cramer-von Mises minimum distance estimators}

The CV estimator is a type of minimum distance estimators which is based on the difference between the estimate of the cdf and the empirical cdf (see D'Agostino and Stephens [4] and Luceño [13]). The CV estimators are obtained by minimizing

$$
C(\varpi)=\frac{1}{12 n}+\sum_{i=1}^{n}\left(\frac{\alpha^{\frac{\gamma+\beta-\left(\gamma+\beta+\gamma \beta x_{(i)}\right) e^{-\gamma x_{(i)}}}{\gamma+\beta}}-1}{\alpha-1}-\frac{2 i-1}{2 n}\right)^{2} .
$$

MacDonald [14] mentioned that the choice of CV method type minimum distance estimators providing empirical evidence that the bias of the estimator is smaller than the other minimum distance estimators.

\subsection{Simulation study}

A simulation study is conducted to evaluate and compare the behavior of the estimates with respect to their mean square errors (MSEs), and absolute biases (ABs). We generate 1000 random sample $X_{1}, \ldots, X_{n}$ of sizes $n=10,30$, and 100 from APTEE distribution. Four choices sets of parameters are considered as:

$$
\begin{array}{ll}
\text { set } 1 \equiv(\alpha=0.5, \gamma=0.8, \beta=2), & \text { set } 2 \equiv(\alpha=0.3, \gamma=0.8, \beta=2), \\
\text { set } 3 \equiv(\alpha=1.25, \gamma=1.75, \beta=0.3), & \text { set } 4 \equiv(\alpha=1.5, \gamma=1.75, \quad \beta=0.3) .
\end{array}
$$

The ML, CV, and PR estimates of $\alpha, \gamma$, and $\beta$ are computed. Then, the ABs and MSEs of the estimates of the unknown parameters are computed. Simulated outcomes are listed in Table 4 and the following observations are detected. 
1. The ABs and MSEs decrease as sample sizes increase for all estimates (see Figures 3 and 4).

2. The ABs and MSEs of ML estimates, for $\alpha$ and $\gamma$ estimates are smaller than the corresponding for $\beta$ (see Table 4).

3. For fixed values of $\gamma, \beta$, and as the values of $\alpha$ decrease, the ABs and MSEs of all estimates are decreasing, in approximately most of situations (see Table 4).

4. The MSEs of the ML estimates of $\gamma$ and $\beta$ take the smallest value among the corresponding MSEs for the other methods in almost all of the cases (see Table 4).
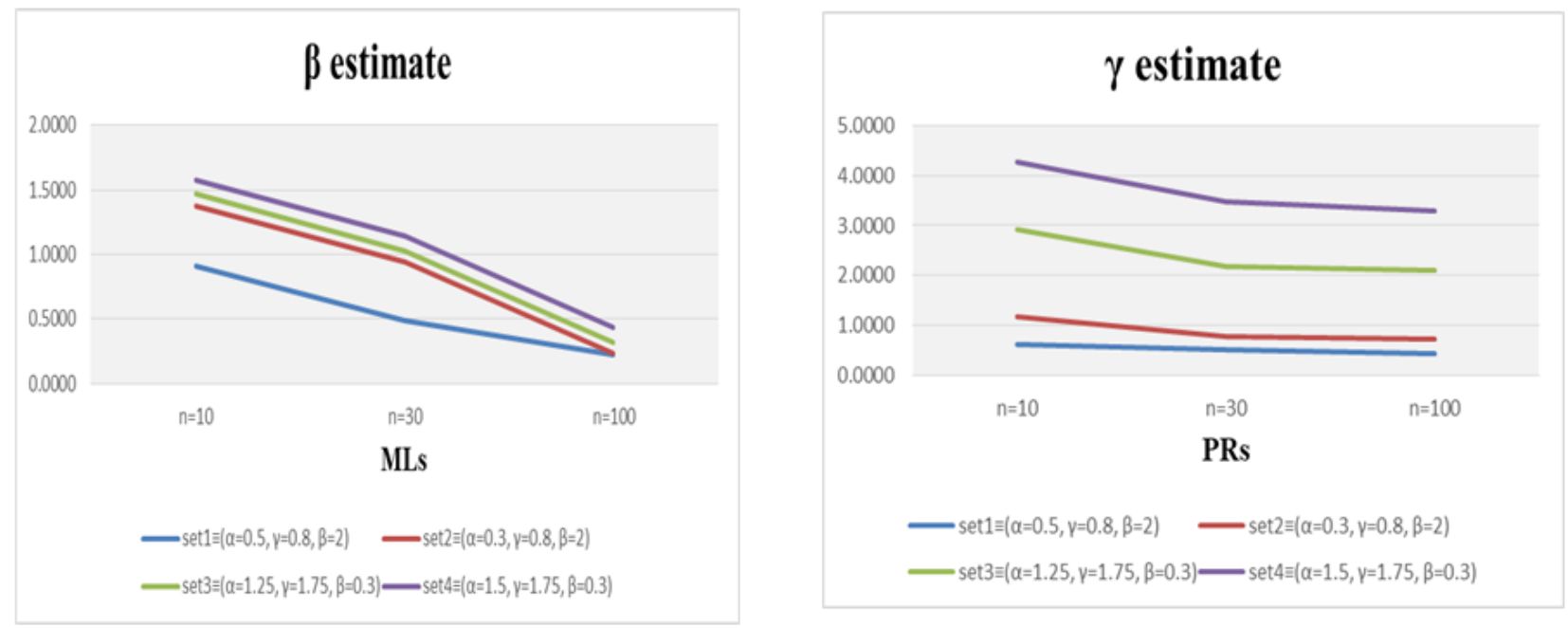

Figure 3: MSE of $\beta$ for the ML estimates for all values of Figure 4: MSE of $\gamma$ for the PR estimates for all set values of parameters. parameters.

5. As it seems from Figure 5, the MSEs of the ML estimates of $\alpha$ take the smallest values corresponding to the other estimates of all methods for the same sample size and for all values of parameters. Also, from Figure 5 the MSEs of ML of $\alpha$ for all set of parameters have the smallest values for the same sample size. Generally, the set 1 of parameters has the smallest MSEs corresponding to other set of parameters.

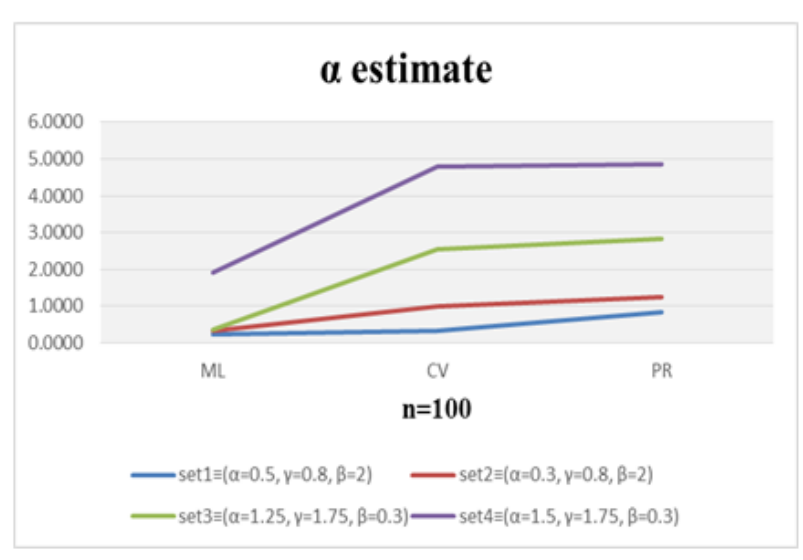

Figure 5: MSEs of $\alpha$ for ML, CV and PR estimates.

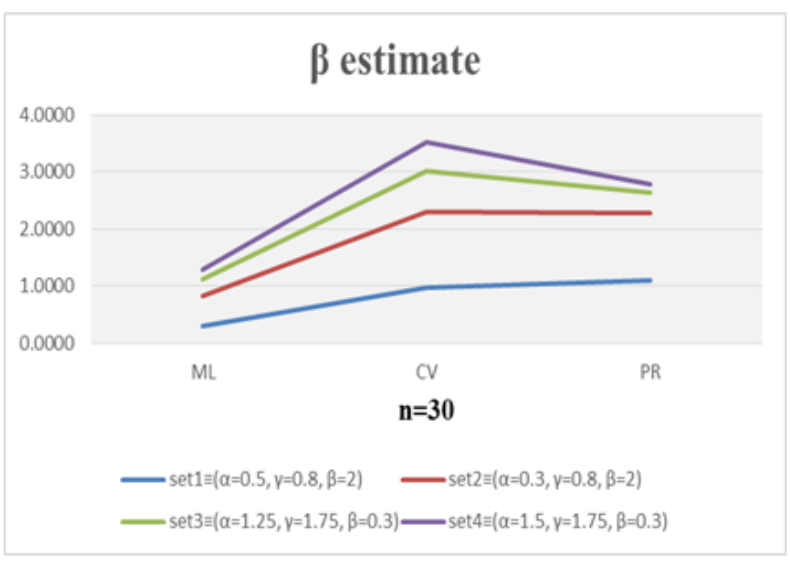

Figure 6: $\mathrm{ABs}$ of $\beta$ for $\mathrm{ML}, \mathrm{CV}$ and PR estimates.

6. As it seems from Figure 6, the ABs of the ML of $\beta$ take the smallest values corresponding to the other estimates of all methods for the same sample size. Also the ABs of $\beta$ for the three sets of 
parameters take the smallest values for the same sample size. Generally, the set 1 of parameters gives the smallest $\mathrm{ABs}$ for different $\beta$ estimates corresponding to other sets of parameters.

Table 4: MSEs and ABs of APTEE distribution for ML, PR, and CV estimates.

\begin{tabular}{|c|c|c|c|c|c|c|c|c|c|c|c|c|c|c|}
\hline \multirow{2}{*}{$n$} & \multirow{2}{*}{ Methods } & \multirow{2}{*}{ Properties } & \multicolumn{3}{|c|}{ set $1 \equiv(\alpha=0.5, y=0.8, \beta=2)$} & \multicolumn{3}{|c|}{ set $2 \equiv(\alpha=0.3, \gamma=0.8, \beta=2)$} & \multicolumn{3}{|c|}{ set $\beta=(\alpha=1.25, \gamma=1.75, \beta=0.3)$} & \multicolumn{3}{|c|}{ setf $=(\alpha=1.5, F 1.75, \beta=0.3)$} \\
\hline & & & $\alpha$ & $\bar{p}$ & $\beta$ & $\alpha$ & y & $\beta$ & $\alpha$ & $y$ & $\beta$ & $\alpha$ & $\bar{y}$ & $\bar{\beta}$ \\
\hline \multirow{6}{*}{10} & \multirow[b]{2}{*}{$\mathrm{ML}$} & MSE & 0.2500 & .0270 & 9070 & 0.0900 & 0.0210 & 0.4690 & 0.0989 & 1.7244 & 0.0900 & 0.1586 & 1.6688 & 0.1084 \\
\hline & & $A B$ & 0.5000 & 0.0060 & 0.9210 & 0.3000 & 0.1220 & 0.5930 & 0.1122 & 1.3120 & 0.5283 & 1.2788 & 1.7201 & 0.3292 \\
\hline & \multirow[b]{2}{*}{ C } & MSE & 0.5854 & 0.6400 & 0.9761 & 0.7437 & 0.6400 & 1.8335 & 1.5625 & 1.7497 & 1.4095 & 2.3374 & 1.9424 & 0.3643 \\
\hline & & $\overline{A B}$ & 0.5921 & 0.7995 & 0.9065 & 1.3205 & 0.7999 & 0.7690 & 1.2500 & 1.3186 & 0.9798 & 1.5000 & 1.1863 & 0.1124 \\
\hline & \multirow{2}{*}{ PR } & MSE & 0.9500 & 0.5353 & 1.6057 & 0.5299 & 1.5606 & 1.6342 & 1.6211 & 2.9796 & 0.5300 & 2.3240 & 2.9794 & 0.4922 \\
\hline & & $A B$ & 0.9649 & 0.6302 & 1.2412 & 0.7145 & 0.5566 & 1.2523 & 1.2500 & 1.7258 & 0.5278 & 1.5000 & 1.3627 & 0.4818 \\
\hline \multirow{6}{*}{30} & \multirow{2}{*}{$\mathbb{M}$} & MSE & 0.2500 & 0.5306 & 0.4910 & 0.0900 & 0.0140 & 0.4480 & 0.0798 & 1.3758 & 0.0900 & 1.5723 & 1.5890 & 0.1083 \\
\hline & & $A B$ & 5000 & 290 & 30 & 0.3000 & 090 & 90 & 1187 & 1.3650 & 0. & 1.5000 & 1.7212 & 0.1716 \\
\hline & \multirow{2}{*}{$\mathrm{CV}$} & MBE & 0.4020 & 0.6392 & 0.9714 & 0.6705 & 0.6399 & 1.7996 & 1.5620 & 1.7452 & 0.7224 & 2.2500 & 1.8990 & 0.3110 \\
\hline & & $A B$ & 0.5770 & 0.7978 & 0.9692 & 0.7958 & 0.7999 & 1.3395 & 1.2457 & 1.3178 & 0.7093 & 1.5000 & 1.3738 & 0.5124 \\
\hline & \multirow{2}{*}{ PR } & MBE & 0.8665 & 0.4280 & 1.5750 & 0.4589 & 0.8838 & 1.6149 & 1.6210 & 2.9784 & 0.2826 & 2.3205 & 1.8732 & 0.0322 \\
\hline & & $\overline{A B}$ & 0.8765 & 0.5306 & 1.0944 & 0.6553 & 0.2642 & 1.1949 & 1.2730 & 1.3758 & 0.3499 & 1.5000 & 1.3047 & 0.1520 \\
\hline \multirow{6}{*}{100} & \multirow{2}{*}{ M } & MSE & 0.2490 & 0.0070 & 0.2220 & 0.0890 & 0.0010 & 0.0130 & 0.0331 & 1.3279 & 0.0897 & 1.5534 & 1.1480 & 0.1078 \\
\hline & & $\overline{A B}$ & 0.4990 & 0.0070 & 0.4310 & 0.2990 & 0.0090 & 0.0040 & 0.0636 & 1.1514 & 0.2994 & 1.2077 & 1.3477 & 0.3301 \\
\hline & & MSE & 0.3417 & 0.6391 & 0.9496 & 0.6514 & 0.6036 & 1.6238 & 1.5618 & 1.7386 & 0.2471 & 2.2491 & 1.8742 & 0.2410 \\
\hline & & $\overline{A B}$ & 0.4999 & 0.1389 & 0.6474 & 0.7609 & 0.3595 & 1.2550 & 1.2497 & 1.3151 & 0.3848 & 1.4997 & 1.3648 & 0.1786 \\
\hline & \multirow{2}{*}{ PR } & MSE & 0.8447 & 0.3883 & 1.5554 & 0.4141 & 0.5055 & 0.9356 & 1.5624 & 1.8632 & 0.0600 & 2.0306 & 1.6044 & 0.0225 \\
\hline & & $A B$ & 0.8267 & 0.4290 & 1.1308 & 0.6329 & 0.3079 & 0.8792 & 1.2500 & 1.3650 & 0.2450 & 1.4250 & 1.1800 & 0.1500 \\
\hline
\end{tabular}

\section{Real data illustration}

To illustrate the usefulness of the APTEE model, we provide analysis to two real data sets. The first data set taken from Linhart and Zucchini [12], represents the failure times of air-conditioned system of an airplane. While second data set taken from Aarset [1] represents the failure times of 50 devices. We fit the APTEE distribution and other five competing models namely; alpha power transformed Weibull (APTW) (Nassar et al. [18]), APTL, APTE, Lindley (L), and exponential (E) distributions. The two data sets are recorded in Table 5.

Table 5: The failure times of air-conditioned system of an airplane and Aarset data.

\begin{tabular}{|c|c|}
\hline Data 1 & $\begin{array}{l}23,261,87,7,120,14,62,47,225,71,246,21,42,20,5,12,120, \\
11,3,14,71,11,14,11,16,90,1,16,52,95\end{array}$ \\
\hline Data 2 & $\begin{array}{l}0.1,0.2,1,1,1,1,1,2,3,6,7,11,12,18,18,18,18,18,21,32,36, \\
40,45,46,47,50,55,60,63,63,67,67,67,67,72,75,79,82,82, \\
83,84,84,84,85,85,85,85,85,86,86\end{array}$ \\
\hline
\end{tabular}

The ML estimates along with their standard error (SE) of the model parameters are provided in Tables 6 and 7. In the same tables, the analytical measures including; minus log-likelihood(-log L) Kolmogorov- 
Smirnov (KS) test statistic, Akaike information Criterion (AIC), corrected Akaike information criterion (CAIC), Bayesian information criterion (BIC) and Hannan-Quinn information criterion (HQIC) are presented.

Table 6: Analytical results of the APTEE model and other competing models for data 1.

\begin{tabular}{|c|c|c|c|c|c|c|c|}
\hline Model & ML Estimates (SE) & -Log L & AIC & BIC & CAIC & HQIC & KS \\
\hline APTEE & $\begin{array}{c}\hat{\alpha}=0.161(0.282) \\
\hat{\beta}=2.01 \times 10^{-4}(0.024) \\
\hat{\gamma}=0.011(0.022)\end{array}$ & $\mathbf{1 7 6 . 6 3 1}$ & $\mathbf{3 5 9 . 2 6 2}$ & $\mathbf{3 5 7 . 6 9 4}$ & $\mathbf{3 6 0 . 1 8 6}$ & $\mathbf{3 6 0 . 6 0 7}$ & $\mathbf{0 . 1 4 6 8 3}$ \\
\hline APTW & $\begin{array}{c}\hat{\alpha}=\begin{array}{c}6.257 \times 10^{-10}\left(9.854 \times 10^{-8}\right) \\
\hat{\theta}=0.509(0.095) \\
\hat{\gamma}=7.168 \times 10^{-3}(0.057)\end{array} \\
\text { APTL }\end{array} \begin{array}{c}\hat{\alpha}=0.1(0.104) \\
\hat{\gamma}=0.024\left(5.127 \times 10^{-3}\right)\end{array}$ & 182.718 & 371.436 & 369.867 & 372.359 & 372.78 & 0.26544 \\
\hline APTE & $\begin{array}{c}\hat{\alpha}=8.688 \times 10^{-10}\left(5.698 \times 10^{-8}\right) \\
\hat{\gamma}=8.536 \times 10^{-4}\left(2.844 \times 10^{-3}\right)\end{array}$ & 177.388 & 370.83 & 369.784 & 371.274 & 371.727 & 0.2803 \\
\hline L & $\hat{\gamma}=7.723 \times 10^{-3}(0.001)$ & 202.837 & 407.674 & 407.151 & 407.817 & 408.123 & 0.663 \\
\hline E & $\hat{\gamma}=4.339 \times 10^{-4}\left(7.922 \times 10^{-5}\right)$ & 233.056 & 468.111 & 467.589 & 468.254 & 468.56 & 0.8929 \\
\hline
\end{tabular}

Table 7: Analytical results of the APTEE model and other competing models for data 2.

\begin{tabular}{|c|c|c|c|c|c|c|c|}
\hline Model & ML Estimates (SE) & -Log L & AIC & BIC & CAIC & HQIC & KS \\
\hline APTEE & $\begin{array}{c}\hat{\alpha}=2.11(1.643) \\
\hat{\beta}=0.021(0.021) \times 10^{-4}(0.024) \\
\hat{\gamma}=0.035\left(6.552 \times 10^{-3}\right)\end{array}$ & $\mathbf{2 8 1 . 4 4 7}$ & $\mathbf{5 6 8 . 8 9 3}$ & $\mathbf{5 6 7 . 9 9}$ & $\mathbf{5 6 9 . 8 1 6}$ & $\mathbf{5 7 1 . 0 7 8}$ & $\mathbf{0 . 1 7 4 1 9}$ \\
\hline APTW & $\begin{array}{c}\hat{\alpha}=8.911(9.1374) \\
\hat{\theta}=0.685(0.128) \\
\hat{\gamma}=0.121(0.078)\end{array}$ & 281.962 & 569.928 & 569.0257 & 570.851 & 572.113 & 0.17423 \\
\hline APTL & $\begin{array}{c}\hat{\alpha}=4.359 \times 10^{-6}\left(6.762 \times 10^{-3}\right) \\
\hat{\gamma}=8.754 \times 10^{-3}\left(6.886 \times 10^{-3}\right)\end{array}$ & 269.747 & 597.495 & 569.892 & 597.939 & 598.951 & 0.2186 \\
\hline APTE & $\begin{array}{c}\hat{\alpha}=4.822 \times 10^{-3}\left(3.528 \times 10^{-6}\right) \\
\hat{\gamma}=1.361 \times 10^{-3}\left(6.19 \times 10^{-3}\right)\end{array}$ & 283.583 & 571.167 & 570.565 & 571.611 & 572.623 & 0.19311 \\
\hline L & $\hat{\gamma}=7.137 \times 10^{-3}(0.00071)$ & 347.347 & 696.694 & 696.393 & 696.837 & 697.422 & 0.8712 \\
\hline E & $\hat{\gamma}=4.204 \times 10^{-4}\left(5.9448 \times 10^{-5}\right)$ & 389.68 & 781.36 & 781.059 & 781.503 & 782.088 & 0.9645 \\
\hline
\end{tabular}

Based on Tables 6 and 7, it is clear that APTEE distribution provides the overall best fit and therefore could be chosen as the more adequate model than other models for explaining the considered data set. More information can be provided in Figures 7 and 9. Also PP-plots are shown in Figures 8 and 10 for both real data.
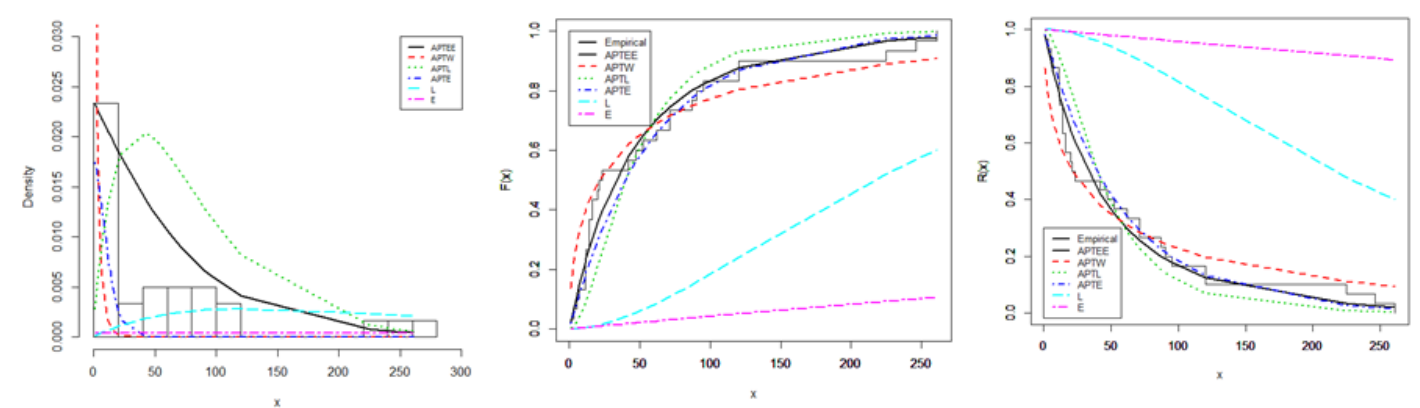

Figure 7: Estimated pdf, cdf, and reliability function of APTEE and other competing models for data 1. 

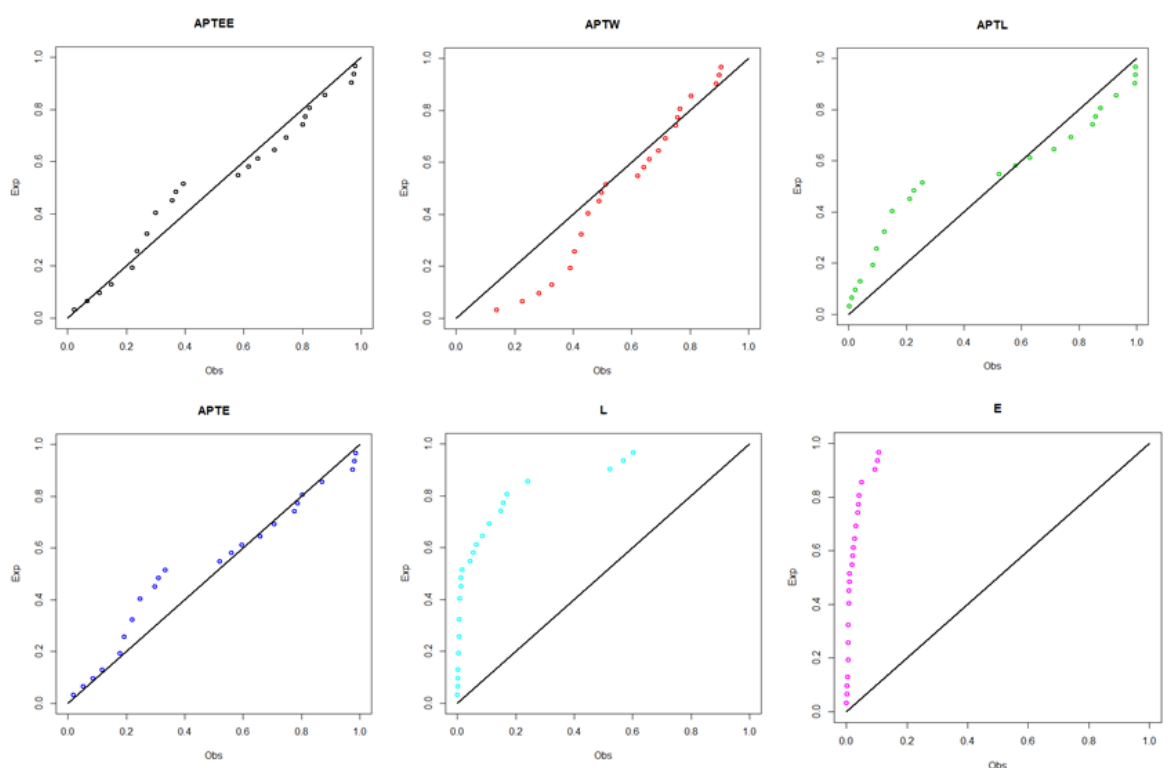

Figure 8: PP plots of APTEE and other competing distributions corresponding to data 1.
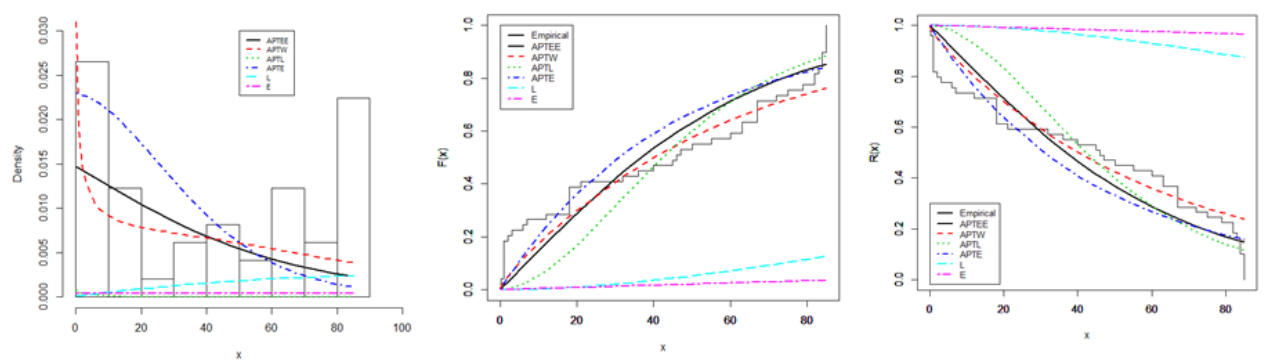

Figure 9: Estimated pdf, cdf and survival function of APTEE and other competing distributions for data 2.
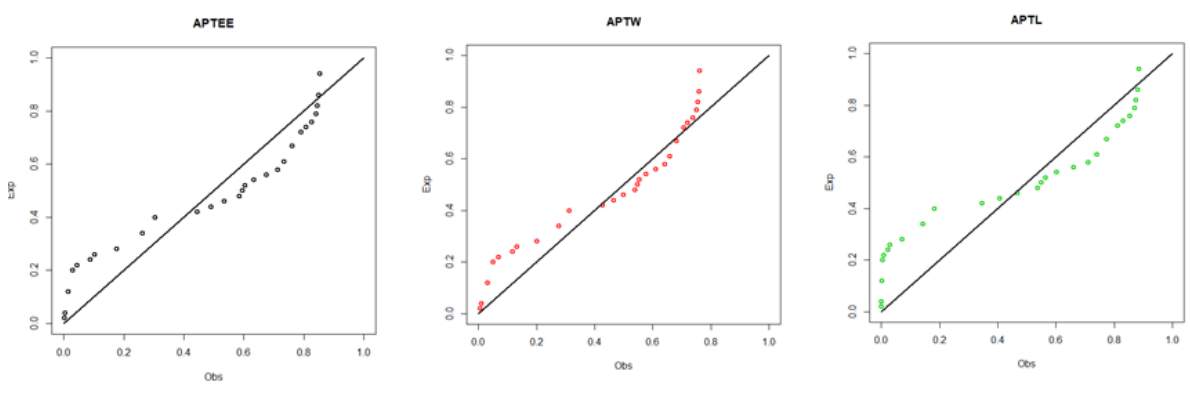

APTE
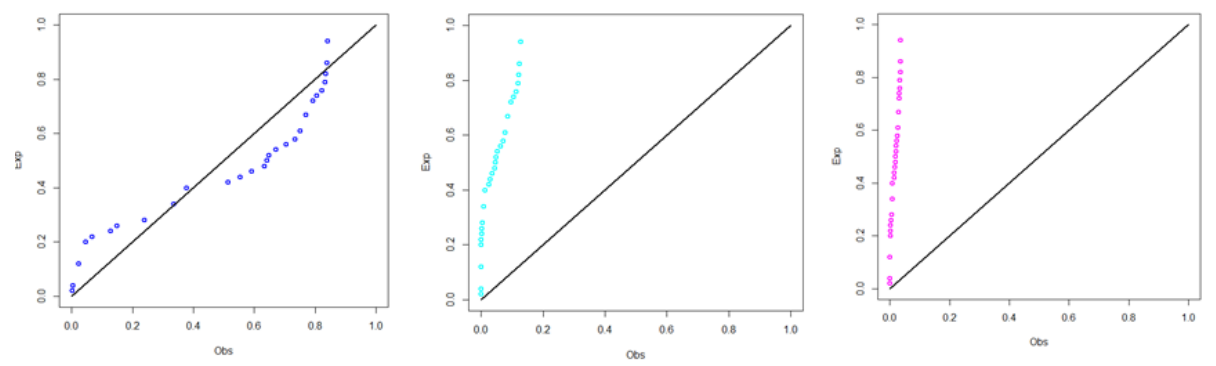

Figure 10: PP plots of APTEE and other competing distributions corresponding to data 2. 
From figures 7-10, we conclude that the APTEE distribution provides better fits then we expect that the proposed model may be an interesting alternative model for a wider range of statistical research.

\section{Concluding remarks}

In this paper, we study the so-called alpha power transformed extended exponential distribution. The APTEE model comprises alpha power transformed exponential, alpha power transformed Lindley, exponential, gamma and Lindley distributions as special sub-models. Some structural properties of the APTEE distribution are derived. Estimation of the population parameters is achieved via three different procedures. Simulation results are carried to assess the accuracy and performance of different estimators. Real data sets are used to illustrate the applications of APTEE distribution.

\section{References}

[1] M. V. Aarset, How to identify a bathtub hazard rate, IEEE Trans. Reliab., 36 (1987), 106-108. 5

[2] G. M. Cordeiro, M. de Castro, A new family of generalized distributions, J. Stat. Comput. Simul., 81 (2011), $883-898$. 1

[3] R. M. Corless, G. H. Gonnet, D. E. G. Hare, D. J. Jeffrey, D. E. Knuth, On the Lambert W function, Adv. Comput. Math., 5 (1996), 329-359. 3.1

[4] R. B. D’Agostino, M. A. Stephens, Goodness-of-Fit Techniques, Marcel Dekker, New York, (1986). 4.3

[5] T. A. N. de Andrade, M. Bourguignon, G. M. Cordeiro, The exponentiated generalized extended exponential distribution, J. Data Sci., 14 (2016), 393-413. 1, 3.1

[6] S. Dey, I. Ghosh, D. Kumar, Alpha power transformed Lindley distribution: Properties and associated inference with application to earthquake data, Ann. Data Sci., 2018 (2018), 1-28. 1, 1

[7] Y. M. Gómez, H. Bolfarine, H. W. Gómez, A new extension of the exponential distribution, Rev. Colombiana Estadıst., 37 (2014), 25-34. 1

[8] R. D. Gupta, D. Kundu, Exponentiated exponential family: An alternative to gamma and Weibull distributions, Biom. J., 43 (2001), 117-130. 1

[9] R. D. Gupta, D. Kundu, Generalized exponential distributions, Aust. N. Z. J. Stat., 41 (1999), 173-188. 1

[10] P. Jodra, Computer generation of random variables with Lindley or Poisson-Lindley distribution via the Lambert W function, Math. Comput. Simulation, 81 (2010), 851-859. 3.1

[11] D. V. Lindley, Fiducial distributions and Bayes' theorem, J. Roy. Statist. Soc. Ser. B, 20 (1958), 102-107. 1

[12] H. Linhart, W. Zucchini, Model Selection, John Wiley \& Sons, New York, (1986). 5

[13] A. Luceño, Fitting the generalized Pareto distribution to data using maximum goodness-of-fit estimators, Comput. Statist. Data Anal., 51 (2006), 904-917. 4.3

[14] P. D. M. MacDonald, Comment on "An estimation procedure for mixtures of distributions" by Choi and Bulgren, J. Roy. Statist. Soc. Ser. B, 33 (1971), 326-329. 4.3

[15] A. Mahadavi, D. Kundu, A new method of generating distribution with an application to exponential distribution, Comm. Statist. Theory Methods, 46 (2017), 6543-6557. 1, 1, 1

[16] F. Merovci, Transmuted exponentiated exponential distribution, Math. Sci. Appl. E-Notes, 1 (2013), 112-122. 1

[17] S. Nadarajah, F. Haghighi, An extension of the exponential distribution, Statistics, 45 (2011), 543-558. 1

[18] M. Nassar, A. Alzaatreh, M. Mead, O. Abo-Kasem, Alpha power Weibull distribution: Properties and applications, Comm. Statist. Theory Methods, 46 (2017), 10236-10252. 5

[19] M. Rasekhi, M. Alizadeh, E. Altun, G. G. Hamedani, A. Z. Afify, M. Ahmad, The modified exponential distribution with applications, Pakistan J. Statist., 33 (2017), 383-398. 1

[20] A. Rényi, On measures of entropy and information, In: Proc. 4th Berkeley Sympos. Math. Statist. and Prob. (University of California Press, Berkeley), 1961 (1961), 547-561. 3.5

[21] M. M. Ristic, N. Balakrishnan, The gamma-exponentiated exponential distribution, J. Stat. Comput. Simul., 82 (2012), 1191-1206. 1

[22] M. Shaked, J. G. Shanthikumar, Stochastic Orders, John Wiley \& Sons, New York, (2007). 3.6 